\title{
Acessibilidade a serviços de saúde bucal por pessoas idosas: uma revisão integrativa
}

\author{
Elderly's accessibility to oral health services: an integrative review
}

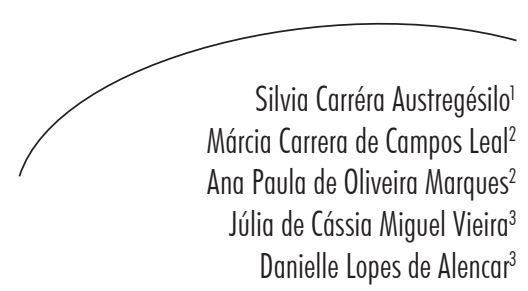

Resumo

Objetivo: Analisar as evidências científicas publicadas relacionadas à acessibilidade de pessoas idosas aos serviços de saúde bucal. Metodologia: Utilizaram-se as bases de dados MEDLINE, LILACS e BBO, empregando como descritores: "idoso", "saúde bucal" e "acesso aos serviços de saúde". A busca ocorreu de forma online em pares, incluindo artigos originais na língua portuguesa, inglesa ou espanhola abordando o tema proposto, publicados no período de janeiro de 2005 a novembro de 2012. Resultados: Mostram estudos em diversos países, os quais referem que a fonte regular de cuidados odontológicos é identificada como fator facilitador para a utilização recente dos serviços. Portanto, apontam a necessidade de ampliar o acesso da população idosa a ações e serviços de saúde bucal, associados principalmente a questões socioeconômicas e culturais. Conclusão: É fundamental garantir o acesso e motivar a procura pelo serviço entre idosos, esclarecendo à população a necessidade e a importância do uso de serviços odontológicos.

\section{Abstract}

Aim: To analyze the scientific evidence published, relating to accessibility of elderly to oral health services. Methodology: Databases MEDLINE, LILACS, BBO were used, employing as descriptors: "elderly", "oral health", "health services accessibility". The search was conducted online in pairs including original articles in Portuguese, English or Spanish on the study theme published between January 2005 and November, 2012. Results: Showed studies in several countries, which refer to the regular source of dental care identified as facilitating factor for the recent use of services. Therefore, they point the need to expand the elderly's access to actions and oral health services, mainly associated socioeconomic and cultural issues. Conclusion: It is essential to ensure access and motivate the demand for service between dentate and edentulous elderly, explaining to the people the need and importance of attending dental services.

\footnotetext{
Universidade Federal de Pernambuco. Programa de Pós-graduação Integrado em Saúde Coletiva. Recife, PE, Brasil

2 Universidade Federal de Pernambuco. Centro de Ciências da Saúde, Departamento de Medicina Social. Recife, PE, Brasil

3 Universidade Federal de Pernambuco. Programa de Pós-graduação em Enfermagem. Recife, PE, Brasil.
}

Palavras-chave: Idoso. Saúde Bucal. Acesso aos Serviços de Saúde.

Key words: Elderly. Oral Health. Health Services Accessibility. 


\section{INTRODUÇÃO}

O processo de envelhecimento humano vivenciado pela população brasileira nos dias atuais pode ser explicado pelos progressos tecnológicos e melhorias nos padrões de saúde da população, com aumento significativo da expectativa de vida, diminuição acentuada das taxas de natalidade, mortalidade infantil e mortalidade por doenças infecciosas. Tal processo tem acarretado aumento considerável da proporção de idosos. ${ }^{1}$

Juntamente com o envelhecimento populacional, observa-se uma transição epidemiológica, caracterizada pelo aumento de doenças crônico-degenerativas em detrimento das infecto-contagiosas, resultando no aumento da demanda dessa população por serviços de saúde. ${ }^{2}$

Dentre os vários aspectos da saúde, a saúde bucal merece atenção especial porque, atualmente, os idosos carregam a herança de um modelo assistencial centrado em práticas curativas e mutiladoras, o que resultou em um quadro atual precário, com ausência de dentes e acúmulo de necessidades de tratamento. ${ }^{1,2}$ Historicamente, os serviços odontológicos não possuem como prioridade a atenção a esse grupo populacional, que, da mesma forma que a população adulta, possui altos níveis de edentulismo e alta prevalência de cárie e de doenças periodontais. ${ }^{2}$

A histórica escassez de atenção odontológica a grupos não escolares resultou na necessidade de formulação de políticas públicas para promoção de saúde bucal para pessoas idosas, para que elas experimentem essa época da vida com qualidade. ${ }^{3}$ Apesar desse panorama de demandas odontológicas acumuladas ao longo da vida, no Brasil é baixo o relato de uso dos serviços odontológicos por idosos, uma vez que $6 \%$ relataram nunca terem utilizado os serviços odontológicos, somente $17 \%$ utilizaram os serviços há menos de um ano, 11\% utilizaram entre um e dois anos e $66 \%$ o fizeram há três ou mais anos. ${ }^{4}$
O uso dos serviços odontológicos, com periodicidade e frequência apropriadas, contribui para a manutenção da saúde bucal, por intermédio de tratamento precoce e prevenção de doenças em todas as idades. ${ }^{3}$ No Brasil, são críticos os indicadores de saúde bucal no que se refere à população idosa. O conhecimento do acesso dessa população aos serviços de odontologia e os fatores relacionados a este acesso são o ponto de partida para se elaborar políticas públicas que possam melhor direcionar estes indivíduos para o uso de serviços de saúde bucal. ${ }^{4,5}$ Portanto, o objetivo deste estudo foi analisar as evidências científicas publicadas que retratam os fatores relacionados à acessibilidade dos idosos aos serviços de saúde bucal.

\section{METODOLOGIA}

Trata-se de uma revisão integrativa, cuja finalidade é reunir e sintetizar resultados de pesquisas sobre um tema delimitado ou questão, de maneira sistemática e ordenada, contribuindo para o aprofundamento do conhecimento do tema investigado. ${ }^{6}$

Para elaboração da presente revisão, foram utilizadas as seguintes etapas: estabelecimento da questão norteadora; seleção dos artigos e estabelecimento dos critérios de inclusão; obtenção dos artigos que constituíram a amostra; avaliação dos artigos; interpretação dos resultados e apresentação da revisão integrativa. ${ }^{7}$ Elaborouse a questão norteadora: quais são as evidências científicas publicadas nos últimos sete anos que abordam os fatores relacionados à acessibilidade dos idosos aos serviços de odontologia?

A pesquisa dos artigos ocorreu em pares, realizada por dois autores do artigo no mês de dezembro de 2012 de forma online, utilizando os descritores em Ciências da Saúde (DeCS/MeSH): idoso, saúde bucal e acesso aos serviços de saúde. As bases de dados eletrônicas empregadas para seleção dos artigos foram: Medical Literature Analysis and Retrieval System Online (MEDLINE), Literatura Latino-Americana e do Caribe em Ciências da Saúde (LILACS) e Biblioteca Brasileira de Odontologia (BBO). 
Os artigos selecionados obedeceram aos seguintes critérios de inclusão: envolver a temática da acessibilidade de idosos aos serviços de odontologia; responder à pergunta norteadora; ser artigo original; ter sido publicado entre os anos 2005 até novembro de 2012; e estar na língua portuguesa, inglesa ou espanhola. Foram excluídas publicações referentes a resumos de congressos, anais, editoriais, comentários e opiniões, artigos de revisão integrativa e bibliográfica, artigos repetidos em uma ou mais bases de dados, os trabalhos que envolviam idosos e adultos na publicação e estudos que consideravam idosos com idade inferior a 60 anos.

Com o intuito de avaliar a qualidade dos estudos, após a seleção da amostra, os artigos selecionados foram submetidos a dois instrumentos por dois autores de forma separada: adaptado do Critical Appraisal Skills Programme (CASP), esse instrumento possui dez itens que são pontuados, incluindo: 1) objetivo; 2) adequação do método; 3) apresentação dos procedimentos teórico-metodológicos; 4) critérios de seleção da amostra, 5) detalhamento da amostra; 6) relação entre pesquisadores e pesquisados; 7) respeito aos aspectos éticos; 8) rigor na análise dos dados; 9) propriedade para discutir os resultados; e 10) contribuições e limitações da pesquisa. Ao final do instrumento, o estudo é classificado em nível A (06 a 10 pontos), significando possuir boa qualidade metodológica e viés reduzido; ou nível B (até 05 pontos), indicando qualidade metodológica satisfatória, porém com tendência a viés.

No presente estudo, optou-se pela utilização dos artigos classificados no nível A. O segundo instrumento foi o critério da Classificação Hierárquica das Evidências para Avaliação dos Estudos, sendo considerados os seguintes níveis: 1) revisão sistemática ou metanálise; 2) ensaios clínicos randomizados; 3) ensaio clínico sem randomização; 4) estudos de coorte e de casocontrole; 5) revisão sistemática de estudos descritivos e qualitativos; e 6) único estudo descritivo ou qualitativo. ${ }^{8}$ Após a seleção da amostra, os artigos foram lidos crítica e exaustivamente.

\section{RESULTADOS}

Realizada a busca conforme os critérios estabelecidos, a amostra foi representada por 11 artigos; a seleção e a exclusão de acordo com cada base de dados estão representadas no quadro 1 . Inicialmente, 18 artigos foram selecionados, porém seis eram repetições em duas bases de dados e, com a aplicação do instrumento da CASP, um artigo foi excluído por estar evidenciado no nível B, totalizando 11 artigos. Em seguida, foi realizada uma apresentação panorâmica geral dos artigos avaliados, divididos em categorias temáticas.

Quadro 1. Relação dos artigos selecionados segundo base de dados. Recife-PE, 2012.

\begin{tabular}{|c|c|c|c|c|c|}
\hline \multicolumn{7}{|c|}{ Artigos } \\
\hline Base de dados & Encontrados & Selecionados & Repetidos & Excluídos & Analisados \\
\hline BBO & 135 & 1 & 1 & 1 & 0 \\
\hline LILACS & 325 & 9 & 6 & 1 & 8 \\
\hline MEDLINE & 5.305 & 8 & 5 & 5 & 3 \\
\hline
\end{tabular}


No quadro 2, segue a descrição dos artigos quanto ao desenho metodológico; nove artigos utilizaram a pesquisa quantitativa; um, a pesquisa quanti-quali; e um artigo era de revisão sistemática.

Quadro 2. Identificação dos artigos segundo título, autores, objetivo(s), principais resultados, ano de publicação. Recife-PE, 2012.

\begin{tabular}{|c|c|c|c|}
\hline Título / Autores & Objetivo(s) & Principais resultados & Ano \\
\hline $\begin{array}{l}\text { A saúde bucal do idoso } \\
\text { brasileiro: revisão } \\
\text { sistemática sobre o } \\
\text { quadro epidemiológico } \\
\text { e acesso aos serviços de } \\
\text { saúde bucal3 } \\
\text { Moreira } R S \text { et al. }\end{array}$ & $\begin{array}{l}\text { Realizar uma revisão } \\
\text { sistemática da literatura, } \\
\text { no período de } 1986 \text { a } 2004, \\
\text { sobre os problemas bucais } \\
\text { mais prevalentes entre os } \\
\text { idosos brasileiros e conhecer } \\
\text { os principais obstáculos no } \\
\text { acesso aos serviços de saúde. }\end{array}$ & $\begin{array}{l}\text { Os valores médios do índice } \\
\text { CPO-D encontrados nos artigos } \\
\text { pesquisados foram de } 25 \text { a } 31 \text {. } \\
\text { Verificou-se grande porcentagem } \\
\text { de indivíduos edêntulos. As } \\
\text { principais barreiras quanto ao } \\
\text { acesso aos serviços odontológicos } \\
\text { foram a baixa escolaridade, a } \\
\text { baixa renda e a escassa oferta de } \\
\text { serviços públicos de atenção à } \\
\text { saúde bucal. }\end{array}$ & 2005 \\
\hline $\begin{array}{l}\text { Idosos de Florianópolis: } \\
\text { autopercepção das } \\
\text { condições de saúde bucal } \\
\text { e utilização de serviços } \\
\text { odontológicos } \\
\text { Benedetti TRB, Mello } \\
\text { ALSF, Gonçalves LHT }\end{array}$ & $\begin{array}{l}\text { Analisar a percepção de saúde } \\
\text { bucal dos idosos participantes } \\
\text { do estudo "Perfil do Idoso do } \\
\text { Município de Florianópolis" } \\
\text { (2004) e o padrão de utilização } \\
\text { de serviços odontológicos, } \\
\text { associando-os com as variáveis } \\
\text { sociodemográficas. }\end{array}$ & $\begin{array}{l}\text { Alta porcentagem de } \\
\text { edentulismo, uso de próteses } \\
\text { e pouca procura por serviços } \\
\text { odontológicos. A consulta } \\
\text { odontológica recente esteve } \\
\text { associada com necessidade de } \\
\text { próteses e encaminhamento } \\
\text { médico. }\end{array}$ & 2007 \\
\hline $\begin{array}{l}\text { Tendência na } \\
\text { utilização de serviços } \\
\text { odontológicos entre } \\
\text { idosos brasileiros e } \\
\text { fatores associados: } \\
\text { um estudo baseado na } \\
\text { Pesquisa Nacional por } \\
\text { Amostra de Domicílios } \\
(1998 \text { e } 2003)^{11} \\
\text { Matos DL, Lima-Costa MF. }\end{array}$ & $\begin{array}{l}\text { Determinar a prevalência e os } \\
\text { fatores associados ao uso de } \\
\text { serviços odontológicos entre } \\
\text { idosos brasileiros em } 1998 \text { e } \\
2003 \text {. }\end{array}$ & $\begin{array}{l}\text { No período considerado, a } \\
\text { prevalência de visitas ao dentista } \\
\text { há }<1 \text { ano aumentou de } 13,2 \\
\text { para } 17,4 \% \text {. Características de } \\
\text { predisposição (sexo, idade e } \\
\text { escolaridade), de necessidade } \\
\text { (percepção da saúde geral) e } \\
\text { de facilitação (renda domiciliar } \\
\text { per capita, filiação a plano de } \\
\text { saúde, situação rural/urbana } \\
\text { do domicílio e macrorregião } \\
\text { de residência) apresentaram } \\
\text { associações independentes e } \\
\text { significantes com o uso de } \\
\text { serviços odontológicos. }\end{array}$ & 2007 \\
\hline
\end{tabular}




\begin{tabular}{|c|c|c|c|}
\hline Título / Autores & Objetivo(s) & Principais resultados & Ano \\
\hline $\begin{array}{l}\text { Uso de serviços } \\
\text { odontológicos entre } \\
\text { idosos brasileiros }{ }^{10} \\
\text { Martins AMEBL, Barreto } \\
\text { SM, Pordeus IA. }\end{array}$ & $\begin{array}{l}\text { Investigar a utilização de } \\
\text { serviços odontológicos e seus } \\
\text { determinantes entre idosos } \\
\text { brasileiros. }\end{array}$ & $\begin{array}{l}\text { O uso de serviços odontológicos } \\
\text { foi menor entre os que mais } \\
\text { necessitavam. Diferentes fatores } \\
\text { estiveram associados ao uso } \\
\text { entre dentados e edentados. } \\
\text { Apenas a escolaridade, o acesso a } \\
\text { informações sobre saúde bucal e } \\
\text { a dor nos dentes ou gengiva nos } \\
\text { últimos três meses foram comuns } \\
\text { aos dois estratos. }\end{array}$ & 2007 \\
\hline $\begin{array}{l}\text { Acesso aos serviços } \\
\text { odontológicos e } \\
\text { motivos da procura por } \\
\text { atendimento por pacientes } \\
\text { idosos em Campina } \\
\text { Grande, } \text { PB }^{18} \\
\text { Costa IMD da, Maciel SML, } \\
\text { Cavalcanti AL }\end{array}$ & $\begin{array}{l}\text { Avaliar o acesso aos serviços } \\
\text { odontológicos e os motivos da } \\
\text { procura por atendimento por } \\
\text { idosos de Campina Grande, } \\
\text { PB. }\end{array}$ & $\begin{array}{l}\text { O serviço público foi o local de } \\
\text { atendimento para } 56,5 \% \text { dos } \\
\text { idosos e a dor se constituiu no } \\
\text { principal motivo para a consulta } \\
(48,4 \%) \text {. Grande parte havia ido } \\
\text { ao cirurgião-dentista há três anos } \\
\text { ou mais (69,4\%). A necessidade } \\
\text { de tratamento foi referida por } \\
71 \% \text { da amostra. }\end{array}$ & 2008 \\
\hline $\begin{array}{l}\text { Uso de serviços } \\
\text { odontológicos por rotina } \\
\text { entre idosos brasileiros: } \\
\text { Projeto SBBrasil }{ }^{4} \\
\text { Martins AMEBL et al. }\end{array}$ & $\begin{array}{l}\text { Investigar o uso de serviços } \\
\text { odontológicos por rotina entre } \\
\text { idosos brasileiros participantes } \\
\text { do Projeto SBBrasil. }\end{array}$ & $\begin{array}{l}\text { Entre dentados, o uso foi } \\
\text { maior entre aqueles com maior } \\
\text { escolaridade e menor entre } \\
\text { residentes no Norte e Nordeste; } \\
\text { na zona rural; que não usaram } \\
\text { no último ano; que não foram } \\
\text { informados sobre como evitar } \\
\text { problemas bucais; que relataram } \\
\text { dor; que necessitavam de próteses } \\
\text { e de tratamento periodontal; que } \\
\text { autoperceberam sua mastigação } \\
\text { irregular cuja condição bucal } \\
\text { afetava o relacionamento; que } \\
\text { autoperceberam sua fala ruim/ } \\
\text { péssima. Entre edentados, foi } \\
\text { maior entre aqueles com maior } \\
\text { escolaridade e usuários de } \\
\text { serviços pagos. }\end{array}$ & 2008 \\
\hline
\end{tabular}




\begin{tabular}{|c|c|c|c|}
\hline Título / Autores & Objetivo(s) & Principais resultados & Ano \\
\hline $\begin{array}{l}\text { Características associadas } \\
\text { ao uso de serviços } \\
\text { odontológicos entre } \\
\text { idosos dentados e } \\
\text { edentados no Sudeste do } \\
\text { Brasil: Projeto SBBrasil }{ }^{10} \\
\text { Martins AMEBL, Barreto } \\
\text { SM, Pordeus IA. }\end{array}$ & $\begin{array}{l}\text { Investigar as características } \\
\text { associadas ao uso de serviços } \\
\text { odontológicos entre idosos da } \\
\text { Região Sudeste do Brasil. }\end{array}$ & $\begin{array}{l}\text { Entre dentados, o uso foi maior } \\
\text { entre idosos que percebiam a } \\
\text { fala influenciada pela saúde } \\
\text { bucal e menor entre residentes } \\
\text { rurais; com menor renda; que } \\
\text { usaram por problema bucal; que } \\
\text { tinham sextante excluído; que } \\
\text { necessitavam de prótese e que } \\
\text { percebiam a aparência como } \\
\text { péssima. Entre edentados, o } \\
\text { uso foi maior entre idosos com } \\
\text { 5-8 anos de escolaridade; que } \\
\text { relatavam sensibilidade dolorosa; } \\
\text { que percebiam a aparência como } \\
\text { péssima; e menor entre idosos } \\
\text { que usaram por problema bucal } \\
\text { e percebiam o relacionamento } \\
\text { afetado pela condição bucal. }\end{array}$ & 2008 \\
\hline $\begin{array}{l}\text { Oral health and access to } \\
\text { dental care: a qualitative } \\
\text { investigation among older } \\
\text { people in the community }{ }^{13} \\
\text { Slack-Smith et al. }\end{array}$ & $\begin{array}{l}\text { Explorar crenças e atitudes } \\
\text { de idosos em relação à saúde } \\
\text { bucal e ao acesso e utilização } \\
\text { de serviços odontológicos. }\end{array}$ & $\begin{array}{l}\text { Cinco temas principais - a } \\
\text { necessidade de informação e } \\
\text { conhecimento; acessibilidade aos } \\
\text { serviços; custo e acessibilidade } \\
\text { aos cuidados orais; medo e } \\
\text { ansiedade em relação a visitas } \\
\text { ao consultório e relacionamento } \\
\text { com o dentista. Atitudes } \\
\text { e comportamentos foram } \\
\text { considerados lentos para } \\
\text { mudanças neste grupo etário. }\end{array}$ & 2009 \\
\hline $\begin{array}{l}\text { Determinantes individuais } \\
\text { da utilização de serviços } \\
\text { odontológicos por adultos } \\
\text { e idosos de baixa renda }{ }^{17} \\
\text { Baldani, M.H. et al. }\end{array}$ & $\begin{array}{l}\text { Identificar os fatores } \\
\text { individuais associados } \\
\text { à utilização de serviços } \\
\text { odontológicos por parte de } \\
\text { adultos e idosos de baixa } \\
\text { renda residentes na área de } \\
\text { abrangência da Estratégia } \\
\text { Saúde da Família, em Ponta } \\
\text { Grossa, PR. }\end{array}$ & $\begin{array}{l}\text { Verificou-se elevada prevalência } \\
\text { de problemas bucais } \\
\text { autorreferidos e de perdas } \\
\text { dentárias. Cerca de } 40 \% \text { dos } \\
\text { adultos e } 67 \% \text { dos idosos não iam } \\
\text { ao dentista há mais de três anos. } \\
\text { Indivíduos que não residiam em } \\
\text { domicílios próprios realizavam } \\
\text { higiene bucal com menor } \\
\text { frequência e utilizavam próteses } \\
\text { totais apresentaram maiores } \\
\text { chances de haver utilizado os } \\
\text { serviços odontológicos há mais } \\
\text { tempo. O fato de possuir um } \\
\text { dentista regular foi identificado } \\
\text { como fator de proteção na } \\
\text { análise. }\end{array}$ & 2010 \\
\hline
\end{tabular}




\begin{tabular}{|c|c|c|c|}
\hline Título / Autores & Objetivo(s) & Principais resultados & Ano \\
\hline $\begin{array}{l}\text { Older Australian } \\
\text { women's use of dentists: } \\
\text { a longitudinal analysis } \\
\text { over six years }{ }^{14} \\
\text { Sibbritt DW, Byles JE, } \\
\text { Tavener } M A \text {. }\end{array}$ & $\begin{array}{l}\text { Identificar os fatores } \\
\text { associados à consulta ao } \\
\text { dentista por mulheres idosas } \\
\text { australianas. }\end{array}$ & $\begin{array}{l}\text { O percentual de mulheres que } \\
\text { consultou um dentista nos anos } \\
\text { de } 1999,2002 \text { e } 2005 \text { foi de } 35 \% \text {, } \\
36 \% \text { e } 37 \% \text {, respectivamente. } \\
\text { Mulheres residentes em áreas } \\
\text { urbanas, não fumantes, não } \\
\text { diabéticas e de melhor saúde } \\
\text { física estavam mais propensas } \\
\text { a consultarem um dentista. } \\
\text { Estavam menos propensas } \\
\text { aquelas com dificuldade de } \\
\text { moradia e baixa renda. }\end{array}$ & 2010 \\
\hline $\begin{array}{l}\text { Density of dental } \\
\text { practitioners and access } \\
\text { to dental care for the } \\
\text { elderly: a multilevel } \\
\text { analysis with a view } \\
\text { on socio-economic } \\
\text { inequality }{ }^{16} \\
\text { L. Lupi-Pegurier et al. }\end{array}$ & $\begin{array}{l}\text { Examinar as relações entre } \\
\text { a densidade de dentista e } \\
\text { fatores socioeconômicos e } \\
\text { demográficos que afetam } \\
\text { o acesso ao atendimento } \\
\text { odontológico pelos idosos. }\end{array}$ & $\begin{array}{l}\text { Baixa renda e falta de seguro } \\
\text { saúde estão associados com } \\
\text { maiores chances de não ter } \\
\text { visitado o dentista, revelando } \\
\text { um acesso de desigual aos } \\
\text { cuidados dentários. A densidade } \\
\text { de dentistas parece ser fator } \\
\text { importante para o acesso } \\
\text { a serviços odontológicos. } \\
\text { Ao considerar as intricadas } \\
\text { relações entre gradiente de } \\
\text { renda e densidade, o último } \\
\text { diminui a desigualdade de } \\
\text { renda relacionada ao acesso aos } \\
\text { serviços odontológicos. }\end{array}$ & 2011 \\
\hline
\end{tabular}




\section{DISCUSSÃO}

Os achados desta revisão retratam o conhecimento dos fatores que determinam o acesso aos serviços de saúde bucal. Todos os artigos analisados enfatizam a importância para subsidiar o planejamento da atenção à saúde do segmento idoso. Esse desafio tem dimensão mundial, uma vez que o envelhecimento populacional é observado em quase todos os países do mundo. A análise do material foi realizada por meio de leitura crítica visando apreender os eixos temáticos referentes ao objetivo proposto.

\section{Condições da saúde bucal de idosos}

Os estudos apontam um grande número de idosos edentados; $;^{3-9}$ além de apresentarem a percepção da saúde bucal como péssima, ${ }^{10}$ tal situação corrobora danos à saúde do idoso não somente no âmbito da saúde bucal, como também no aspecto nutricional e de saúde em geral.

A apresentação do quadro epidemiológico da saúde bucal do idoso brasileiro aparece de maneira bem detalhada e discutida em alguns artigos. ${ }^{1-4,9,10}$ Moreira et al. $^{3}$ trazem em sua revisão sistemática o quadro epidemiológico da saúde bucal desses indivíduos, além de seu acesso aos serviços de atenção odontológica, baseandose no período de 1986 a 2004.

Ainda que o processo de envelhecimento, por si, cause na cavidade bucal poucos efeitos desencadeadores de disfunções e incapacidades, expressivo número de estudos revela que, em geral, a condição de saúde bucal dos idosos é deficiente. ${ }^{9}$

Os danos causados pelas doenças bucais aumentam com a idade, comprometem a qualidade de vida e restringem as atividades cotidianas dos indivíduos. No Brasil, o quadro de saúde bucal dos idosos é crítico: em 2003, apenas 10\% tinham mais de 20 dentes na boca, proporção muito inferior à meta proposta pela Organização Mundial da Saúde (OMS), a qual preconizava que $50 \%$ da população idosa deveriam apresentar mais de 20 dentes na boca até o ano $2000 .{ }^{4,9,11}$

Com a realização do primeiro levantamento epidemiológico em saúde bucal de 1986, foram identificados declínio de presença de dentes hígidos com o avançar da idade, alto nível de edentulismo e poucas pessoas não edêntulas e isentas de problemas periodontais. ${ }^{3,4,9}$

A experiência seguinte foi a Pesquisa Nacional de Saúde Bucal - Projeto SBBrasil, realizada em 2003 e 2010. Em 2003, o índice CPOD (a soma de dentes cariados, perdidos e obturados por cárie num indivíduo) apresentou expressivo incremento com o avançar da idade, e na faixa etária dos 65 aos 74 anos alcançou 27,79, fato explicado em grande parte pelo componente "perdido" em 92\%. Somente 10\% dos idosos conservavam ainda 20 ou mais dentes. Nesse mesmo levantamento, 4,3\% dos idosos consideravam sua saúde bucal ótima; $46 \%$, boa; e $27,5 \%$, regular. ${ }^{3,4,9}$

A edição mais recente dessa pesquisa, em 2010, apesar de não ter sido apresentada em nenhum dos artigos selecionados, apresenta resultados semelhantes: o índice CPOD para indivíduos na faixa etária de 65 aos 74 anos foi de 27,53, valor semelhante ao de 2003, também explicado principalmente pelo componente "perdido". Em relação à autopercepção de sua saúde bucal, $8,1 \%$ consideravam-se muito satisfeitos; $44,5 \%$, satisfeitos; e 17,3, nem satisfeitos nem insatisfeitos. $^{12}$

Diante do novo quadro demográfico, as significativas mudanças no padrão de incidência e prevalência de doenças bucais exigem a concepção e implantação de políticas que orientem os serviços de saúde bucal a honrar sua missão de promover a saúde do idoso.?

Inúmeros fatores podem contribuir para a manutenção da saúde bucal do indivíduo idoso, pois o fato de haver algum tipo de comprometimento físico e de saúde pode 
limitar o acesso ao serviço odontológico, ${ }^{13,14}$ principalmente se fatores socioeconômicos fizerem parte da realidade do idoso.

\section{Fatores associados a0 acesso aos serviços de saúde bucal}

Um dos principais fatores de execução de serviços de saúde são as barreiras de acesso. A principal barreira está relacionada à ausência de recursos humanos, além das barreiras geográficas, financeiras, organizacionais, informacionais, culturais, que expressam o tipo da oferta que, de modo interligado, agem promovendo ou dificultando a possibilidade de essas pessoas utilizarem os serviços de saúde. ${ }^{15}$

É importante ressaltar que alguns aspectos que influenciam o acesso e a utilização dos serviços de saúde podem estar profundamente imbricados, como a escolaridade e a renda.,3,4, 9-11,13 Os achados demonstram que em relação ao uso dos serviços odontológicos, o acesso é prejudicado principalmente por barreiras financeiras, ${ }^{1,3,11,13-15}$ tais como baixa renda, dificuldade de moradia, além de fatores culturais associados ao medo e ansiedade..$^{13}$ Tal quadro direciona ainda para a redução da procura e/ou demora dos serviços odontológicos, sobretudo por parte dos mais necessitados desse serviço. ${ }^{9-11,16-18}$

Dificuldades financeiras constituem importante aspecto de obstáculo na utilização dos serviços de saúde bucal observada não só em países em desenvolvimento, como o Brasil, mas também em países como a Austrália e França. ${ }^{13,16}$ No artigo desenvolvido por SlackSmith et al., ${ }^{13}$ entre as barreiras de acesso aos serviços mencionadas pelos idosos participantes do estudo, estavam o custo pelo tratamento, além da distância dos serviços a suas residências. Na França, baixa renda e ausência de seguro saúde complementar estavam associadas a maior probabilidade de não realizar visitas a dentistas, revelando alta iniquidade de acesso aos serviços de saúde bucal. ${ }^{16}$

Outro aspecto importante relaciona-se a informação e escolaridade: a primeira é descrita nos estudos como empecilho para uso dos serviços odontológicos e saúde bucal em geral, ${ }^{1,10,13}$ devido ao déficit informacional voltado às populações ou pela escassez dos serviços. ${ }^{3}$ Entretanto, a baixa escolaridade também está associada ao menor acesso aos serviços. ${ }^{1,3,11,13}$

De uma maneira geral, os fatores associados ao uso de serviços odontológicos por idosos brasileiros foram semelhantes nos estudos realizados com base em dados secundários (PNAD 1998 e SBBrasil 2003), incluindo as mesmas características de predisposição, necessidade e facilitação. ${ }^{1,4,9,11}$

Diversos são os fatores que interferem no acesso aos serviços de saúde bucal por idosos, sendo a idade importante fator na determinação da menor frequência de visitas ao dentista. $\mathrm{O}$ comportamento das idades mais velhas em visitar menos ou não visitar o dentista pode ser explicado pela alta prevalência da perda de dentes naturais entre idosos brasileiros e pela dificuldade de acesso aos serviços odontológicos., ${ }^{3,411}$ A autoavaliação da saúde como pior entre os idosos pode indicar declínio real da saúde geral, levando à menor capacidade para procurar os serviços odontológicos e/ou a considerar a consulta ao dentista como menos importante, devido aos outros problemas de saúde. ${ }^{10}$

Entre dentados, os fatores associados foram: local de residência, renda per capita, autopercepção da fala, saúde periodontal e necessidade de prótese. Já entre os edentados, os fatores foram: escolaridade, autopercepção do relacionamento e dor de dente ou gengiva nos últimos seis meses. ${ }^{10}$

Pode-se sugerir que a acessibilidade aos serviços odontológicos pode ser influenciada por diferentes prismas de observação, desde os elementos limitadores geográficos, físicos e operacionais quanto à cobertura assistencial, à escassa oferta de serviços públicos de atenção à saúde bucal voltados à população idosa brasileira e às possíveis "barreiras veladas" de acesso.

Destaca-se que, provavelmente, a limitação do estudo deve estar atrelada às necessidades 
de o profissional desenvolver competências relacionadas à capacidade de analisar criticamente o contexto da prática, transformando sua dúvida em questão de pesquisa. Portanto, é necessário ter conhecimento sobre as diversas fontes de informação, delineamento e metodologia da pesquisa, para elaborar uma revisão integrativa que proporcione a síntese de conhecimento e a incorporação da aplicabilidade dos resultados de pesquisas na prática.

As limitações se relacionam também ao uso exclusivo dos descritores DeCS e MeSH para localizar os artigos nas bases de dados eletrônicas, o que pode explicar a recuperação de número limitado de estudos no período determinado, bem como a inclusão de estudos acessados por meio eletrônico, o que restringiu o acesso ao total de estudos selecionados para a revisão.

\section{CONCLUSÃO}

Com base nos artigos utilizados nesta revisão integrativa, que teve por objeto de investigação a acessibilidade aos serviços de saúde bucal por idosos, é possível evidenciar um quantitativo considerável de variáveis que influenciam o acesso, sugerindo que o tema é bastante complexo e pluralista, impossível de ser apreendido por uma única perspectiva metodológica. Este aspecto ressalta a necessidade da utilização de diferentes abordagens, a fim de contribuir para o conhecimento das diferentes realidades locais, com a visão focada sobre quais aspectos do objeto o sujeito visa realizar sua pesquisa.

Conclui-se também que são necessários novos estudos mais focados na atenção ao grupo idoso, abordando suas necessidades e especificidades, de modo a traduzir e refletir sobre os motivos que levam à busca por esses serviços. $\mathrm{O}$ acesso aos serviços de saúde bucal pode ser influenciado por diferentes razões, entre as quais a acessibilidade econômica, cultural e funcional. Os fatores sociodemográficos, a percepção da necessidade, bem como crenças e a importância atribuída à saúde bucal podem também exercer forte influência sobre a utilização de serviços odontológicos.

Nesta revisão, sugere-se que a superação das iniquidades sociais e da escassa oferta de serviços possa servir como forma de redução das barreiras e de permitir o aumento da demanda aos serviços de saúde bucal. É fundamental garantir o acesso e motivar o uso entre idosos dentados e edentados, esclarecendo à população sobre a necessidade e a importância do uso de serviços odontológicos.

\section{REFERÊNCIAS}

1. Martins AMEBL, Haikal DS, Pereira SM, Barreto SM. Uso de serviços odontológicos por rotina entre idosos brasileiros: Projeto SB Brasil. Cad Saúde Pública 2008;24(7):1651-66.

2. Viana AAF, Gomes MJ, De Carvalho RB, De Oliveira ERA. Acessibilidade dos idosos brasileiros aos serviços odontológicos. RFO UPF 2010;15(3):319-24.

3. Moreira RS, Nico LS, Tomita NE, Ruiz T. A saúde bucal do idoso brasileiro: revisão sistemática sobre o quadro epidemiológico e acesso aos serviços de saúde bucal. Cad Saúde Pública 2005;21(6):1665-75.

4. Martins AMEBL, Barreto SM, Pordeus IA. Uso de serviços odontológicos entre idosos brasileiros. Rev Panam Salud Publica 2007;22(5):308-16.

5. Almeida CS, Miotto MHMB, Barcellos LA. O Perfil do usuário do serviço odontológico do Município de São Mateus-ES. UFES Rev Odontol 2007;9(2):8-15.

6. Mendes KDS, Silveira RCCP, Galvão CM. Revisão integrativa: método de pesquisa para a incorporação de evidências na saúde e na enfermagem. Texto \& Contexto Enferm 2008;17(4):758-64.

7. Beya S, Nicoll LH. Writing an integrative review. AORN J 1998;67(4):877-80.

8. Stillwell SB, Fineout-Overholt E, Melnyk BM, Williamson KM. Evidence-based practice: Step by Step. Am J Nurs 2010;110(5):41-7

9. Benedetti TRB, Mello ALSF, Gonçalves LHT. Idosos de Florianópolis: autopercepção das condições de 
saúde bucal e utilização de serviços odontológicos. Ciênc Saúde Coletiva 2007;12(6):1683-90.

10. Martins AMEBL, Barreto SM, Pordeus IA. Características associadas ao uso de serviços odontológicos entre idosos dentados e edentados no sudeste do Brasil: Projeto SB Brasil. Cad Saúde Pública 2008;24(1):81-92.

11. Matos DL, Lima-Costa MF. Tendência na utilização de serviços odontológicos entre idosos brasileiros e fatores associados: um estudo baseado na Pesquisa Nacional por Amostra de Domicílios (1998 e 2003). Cad Saúde Pública 2007;23(11):2740-48.

12. Brasil. Ministério da Saúde, Secretaria de Atenção à Saúde, Departamento de Atenção Básica, Coordenação Geral de Saúde Bucal. SB Brasil 2010: Pesquisa Nacional de Saúde Bucal: Resultados Principais [Internet]. Brasília, DF: Ministério da Saúde; 2011 [acesso em 07 jan 2013]; 92 p. Disponível em: http://189.28.128.100/dab/docs/geral/ projeto_sb2010_relatorio_final.pdf.

13. Slack-Smith L, Lange A, Paley G, O'Grady M, French D, Short L. Oral health and access to dental care: a qualitative investigation among older people in the community. Gerodontology 2010;27(2):104-13.
14. Sibbritt DW, Byles JE, Tavener MA. Older Australian women's use of dentists: a longitudinal analysis over 6 years. Australas J Ageing 2010;9(1):14-20.

15. Travassos C, Castro MSM. Determinantes e desigualdades sociais no acesso e na utilização de serviços de saúde. In: Giovanella L, Escorel S, Lobato LVC, Noronha JC, De Carvalho AI, organizadores. Políticas e Sistemas de Saúde no Brasil. $2^{\mathrm{a}}$ ed. Rio de Janeiro: Fiocruz; 2009. p. 219-21.

16. Lupi-Pegurier L, Clerc-Urmes I, Abu-Zaineh M, Paraponaris A, Ventelou B. Density of dental practitioners and access to dental care for the elderly: a multilevel analysis with a view on socioeconomic inequality. Health Policy 2011;103(2-3):160-7.

17. Baldani MH, Brito WH, Lawder JAC, Mendes YBE, Da Silva FFM, Antunes JLF. Determinantes individuais da utilização de serviços odontológicos por adultos e idosos de baixa renda. Rev Bras Epidemiol 2010;13(1):150-62.

18. Costa IMD, Maciel SML, Cavalcanti AL. Acesso aos serviços odontologicos e motivos da procura por atendimento por pacientes idosos em Campina Grande - PB. Odontol Clín-Cient 2008;7(4):331-35 